\title{
Maurice HALBWACHS
}

Sociologue français, 1877-1945

(1947)

\section{"La mémoire collective et le temps”}

Un document produit en version numérique par Jean-Marie Tremblay, bénévole, professeur de sociologie au Cégep de Chicoutimi

Courriel: jean-marie_tremblay@uqac.ca

Site web pédagogique : http://www.uqac.ca/jmt-sociologue/

Dans le cadre de la collection: "Les classiques des sciences sociales"

Site web: http://classiques.uqac.ca/

Une collection développée en collaboration avec la Bibliothèque

Paul-Émile-Boulet de l'Université du Québec à Chicoutimi

Site web: http://bibliotheque.uqac.ca/ 
Cette édition électronique a été réalisée par Jean-Marie Tremblay, bénévole, professeur de sociologie au Cégep de Chicoutimi à partir de :

Maurice Halbwachs [Sociologue français, 1877-1945]

\section{“La mémoire collective et le temps”.}

Un article publié dans la revue Cahiers internationaux de sociologie, vol. 101, 1996, pp. 45-65. Paris: Les Presses universitaires de France. Publication originale: Cahiers internationaux de sociologie, 1947.

Polices de caractères utilisée :

Pour le texte: Times New Roman, 14 points.

Pour les citations : Times New Roman, 12 points.

Pour les notes de bas de page : Times New Roman, 12 points.

Édition électronique réalisée avec le traitement de textes Microsoft Word 2004 pour Macintosh.

Mise en page sur papier format

LETTRE (US letter), 8.5'” x 11'’)

Édition numérique réalisée le 26 juillet 2006 à Chicoutimi, Ville de Saguenay, province de Québec, Canada. 


\section{Maurice HALBWACHS (1947)}

Sociologue français, 1877-1945

\section{"La mémoire collective et le temps"}

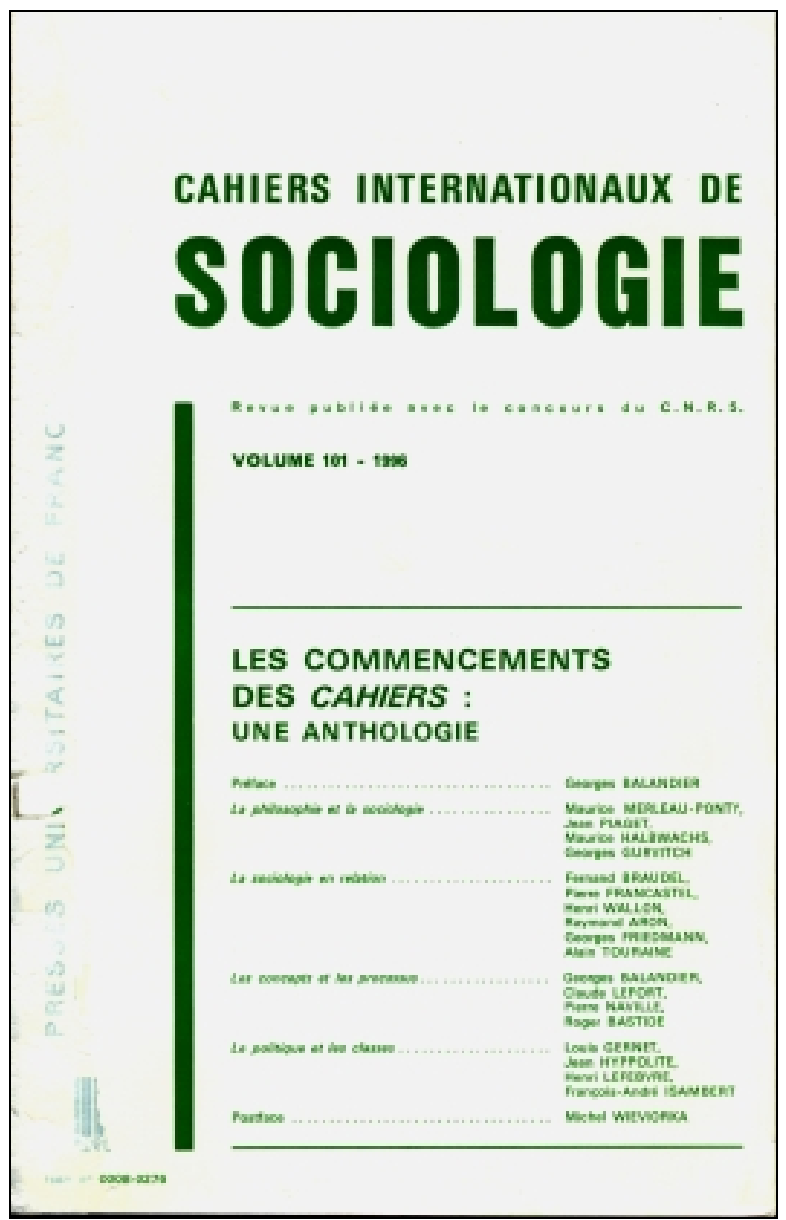

Un article publié dans la revue Cahiers internationaux de sociologie, vol. 101, 1996, pp. 45-65. Paris: Les Presses universitaires de France. Publication originale: Cahiers internationaux de sociologie, 1947. 
Maurice Hakbwachs (1922 - )

"La mémoire collective et le temps". * 1

Un article publié dans la revue Cahiers internationaux de sociologie, vol. 101, 1996, pp. 45-65. Paris: Les Presses universitaires de France. Publication originale: Cahiers internationaux de sociologie, 1947.

La mémoire collective remonte dans le temps jusqu'à une certaine limite, plus ou moins éloignée d'ailleurs, suivant qu'il s'agit de tel ou tel groupe. Au-delà, elle n'atteint plus les événements et les personnes d'une prise directe. Or, c'est précisément ce qui se trouve au-delà de cette limite qui retient l'attention de l'histoire. On dit quelquefois que l'histoire s'intéresse au passé et non au présent. Mais ce qui est vraiment le passé pour elle, c'est ce qui n'est plus compris dans le domaine où s'étend encore la pensée des groupes actuels. Il semble qu'il lui faille attendre que les groupes anciens aient disparu, que leurs pensées et leur mémoire se soient évanouies, pour qu'elle se préoccupe de fixer l'image et l'ordre de succession des faits qu'elle seule est maintenant capable de conserver. Sans doute, il faut bien l'aider alors de témoignages anciens dont la trace subsiste dans des textes officiels, des journaux du temps, des mémoires écrits par des contemporains. Mais dans le choix qu'il en fait, dans l'importance qu'il leur attribue, l'historien se laisse guider par des raisons qui n'ont rien à voir avec l'opinion d'alors, car cette opinion n'existe plus ; on n'est pas obligé d'en tenir compte ; on n'a pas à craindre qu'elle vous oppose un démenti. Tant il est vrai qu'il ne peut faire son œuvre qu'à condition de se placer délibérément hors du temps vécu par les groupes qui ont assisté aux évé-

Cahiers internationaux de sociologie, II, 1947.

1 L'éminent sociologue français, déporté par les Allemands en juillet 1944 comme impliqué dans une action de résistance, est mort à Buchenwald le 16 mars 1945. 
nements, qui en ont eu le contact plus ou moins direct, et qui peuvent se les rappeler.

Plaçons-nous donc maintenant au point de vue des consciences collectives, puisque c'est le seul moyen, pour nous, de rester dans un temps réel, assez continu pour qu'une pensée puisse en parcourir toutes les parties en demeurant elle-même, et en gardant le sentiment de son unité. Nous avons dit qu'il faut distinguer un certain nombre de temps collectifs, autant qu'il y a de groupes séparés. Nous ne pouvons méconnaître cependant que la vie sociale dans son ensemble et dans toutes ses parties s'écoule dans un temps qui est divisé en années, mois, jours, heures. Il faut bien qu'il en soit ainsi, sans quoi si les durées, dans les divers groupes en lesquels se décompose la société, comportaient des divisions différentes, on ne pourrait établir aucune correspondance entre leurs mouvements. Or, précisément, parce que ces groupes sont séparés l'un de l'autre, que chacun d'eux a son mouvement propre, et que les hommes individuels passent cependant de l'un à l'autre, les divisions du temps doivent être partout assez uniformes. Il doit toujours être possible, quand on est dans un premier groupe, de prévoir à quel moment on entrera dans un second, ce moment se référant bien entendu au temps du second. Mais quand on est dans le premier, on est dans le temps du premier, non dans le temps du second. C'est le problème qui se pose à un voyageur qui doit aller à l'étranger et qui ne dispose pour mesurer le temps que des horloges de son pays. Il sera assuré cependant de ne pas manquer son train, si l'heure est la même dans tous les pays, ou s'il y a entre les heures des divers pays un tableau de correspondance.

Dirons-nous, alors, qu'il y a bien un temps unique et universel auquel se réfèrent toutes les sociétés, dont les divisions s'imposent à tous les groupes et que ce branle commun, transmis à toutes les régions du monde social, rétablit entre elles les communications et rapports que leurs barrières mutuelles tendraient à empêcher ? Mais d'abord la correspondance entre les divisions du temps dans plusieurs sociétés voisines est beaucoup moins exacte que lorsqu'il s'agit d'horaires internationaux de chemins de fer. Cela s'explique d'ailleurs par le fait que les exigences des divers groupes à cet égard ne sont pas les mêmes. Dans la famille, en général, le temps comporte un certain jeu, bien plus qu'au lycée ou à la caserne. Bien qu'un curé doive dire sa messe à 
l'heure, rien n'est prévu quant à la durée exacte de son sermon ; en dehors des cérémonies, auxquelles d'ailleurs ils arrivent souvent en retard, et qu'ils ne suivent pas toujours jusqu'au bout, les fidèles peuvent aller à l'église quand il leur plait, et faire chez eux leurs exercices de prières et de dévotions sans se régler sur l'heure astronomique. Un commerçant doit arriver à l'heure pour ne pas manquer un rendez-vous d'affaires ; mais les achats se distribuent sur toute la journée, et, pour les commandes, les livraisons, s'il y a des délais fixés, c'est en général avec une grande approximation. Il semble, d'ailleurs, qu'on se repose ou qu'on prend sa revanche dans certains milieux de l'exactitude à laquelle on est obligé dans d'autres. Il y a une société dont la matière se renouvelle sans cesse, dont les éléments se déplacent les uns par rapport aux autres continuellement, c'est l'ensemble des hommes qui circulent dans les rues. Or, sans doute, quelques-uns d'entre eux sont pressés, hâtent le pas, regardent leur montre aux abords des gares, à l'arrivée dans les bureaux et à la sortie, mais en général quand on se promène, qu'on flâne, qu'on regarde la devanture des magasins, on ne mesure pas la durée des heures, on ne se soucie pas de savoir quelle heure il est exactement, et quand on doit faire un long trajet pour arriver à peu près à temps, on se guide sur un sentiment vague, comme on se dirige dans une ville sans regarder le nom des rues par une sorte de flair. Puisque, dans les divers milieux, on n'éprouve pas le besoin de mesurer le temps avec la même exactitude, il en résulte que la correspondance entre le temps du bureau, le temps de la maison, le temps de la rue, le temps des visites n'est fixée qu'entre des limites quelquefois assez larges. C'est pourquoi on s'excuse d'arriver en retard à un rendez-vous d'affaires, ou de rentrer chez soi à une heure anormale, en disant qu'on a rencontré quelqu'un dans la rue ; cela revient à réclamer le bénéfice de la liberté avec laquelle on mesure le temps dans un milieu où l'on ne se soucie pas trop à cet égard de l'exactitude.

Nous avons parlé surtout d'heures et de minutes, mais on dit quelquefois à un ami : je viendrai vous voir un de ces jours, la semaine prochaine, dans un mois; quand on revoit un parent éloigne, on compte le nombre d'années a peu près depuis lesquelles on ne s'était pas revu. C'est que ce genre de relation ou de société ne comporte pas une localisation dans le temps plus définie. Ainsi et déjà de ce point de vue, ce n'est pas tout à fait le même temps, mais des temps en correspondance plus ou moins exacte, qu'on trouverait dans nos sociétés. 
Il est vrai que tous s'inspirent d'un même type, et se réfèrent à un même cadre qui pourrait être considéré comme le temps social par excellence. Nous n'avons pas à rechercher quelle est l'origine de la division de la durée en années, mois, semaines, jours. Mais c'est un fait que sous la forme que nous lui connaissons, elle est très ancienne et repose sur des traditions. On ne peut dire, en effet, qu'elle résulte d'un accord conclu entre tous les groupes, ce qui impliquerait qu'à un moment donné, ils suppriment les barrières qui les séparent et se fondent pour quelque temps en une seule société qui aurait pour objet de fixer un système de division de la durée. Mais il est possible, il est sans doute nécessaire, qu'autrefois cette entente se soit réalisée dans une société unique d'où toutes celles que nous connaissons seraient sorties. Supposons qu'autrefois les croyances religieuses aient mis fortement leur empreinte sur les institutions. Peut-être des hommes qui réunissaient en eux les attributs des chefs et des prêtres ont-ils divisé le temps, en s'inspirant à la fois de leurs conceptions religieuses et de l'observation du cours naturel des phénomènes célestes et terrestres. Quand la société politique s'est distinguée du groupe religieux, quand les familles se sont multipliées, elles ont continué à diviser le temps de la même manière que dans la communauté primitive d'où elles sortaient. Maintenant encore, quand des groupes nouveaux se forment, groupes durables ou éphémères, entre gens d'une même profession, d'une même ville ou d'un même village, entre amis en vue d'une oeuvre sociale, d'une activité littéraire ou artistique, ou simplement à l'occasion d'une rencontre, d'un voyage en commun, c'est toujours par séparation d'un ou de plusieurs groupes plus larges et plus anciens. Il est naturel que, dans ces formations nouvelles, on retrouve bien des traits des communautés mères, et que beaucoup de notions élaborées dans celles-ci passent dans celles-là ; la division du temps serait une de ces traditions, dont on ne pouvait se passer, d'ailleurs, car il n'est pas de groupe qui n'ait besoin de distinguer et reconnaître les diverses parties de sa durée. C'est ainsi qu'on retrouve dans les noms des jours de la semaine et des mois bien des traces de croyances et de traditions disparues, qu'on date toujours les années à partir de la naissance du Christ, et que de vieilles idées religieuses sur la vertu du nombre 12 sont à l'origine de la division actuelle du jour en heures, minutes et secondes. 
Cependant de ce que ces divisions subsistent, il ne résulte nullement qu'il y ait un temps social unique, car, en dépit de leur origine commune, elles ont pris une signification très différente dans les divers groupes. Ce n'est pas seulement parce que, comme nous l'avons montré, le besoin d'exactitude à cet égard varie d'une société à l'autre, mais d'abord comme il s'agit d'appliquer ces divisions à des séries d'événements ou de démarches qui ne sont pas les mêmes dans plusieurs groupes, et qui se terminent et recommencent à des intervalles qui ne se correspondent pas d'une société à l'autre, on peut dire que l'on compte le temps à partir de dates différentes dans celle-ci et dans celle-là. L'année scolaire ne commence pas le même jour que l'année religieuse. Dans l'année religieuse, l'anniversaire de la naissance du Christ et l'anniversaire de sa mort et de sa résurrection déterminent les divisions essentielles de l'année chrétienne. L'année laïque commence le premier janvier, mais, suivant les professions et les genres d'activités, elle comporte des divisions très différentes; celles de l'année paysanne se règlent sur le cours des travaux agricoles, déterminé luimême par l'alternance des saisons. L'année industrielle ou commerciale se décompose en périodes où l'on travaille à plein rendement, où les commandes affluent, et en d'autres où les affaires se ralentissent ou s'arrêtent. Ce ne sont d'ailleurs pas les mêmes dans tous les commerces et toutes les industries. L'année militaire se compte tantôt en partant de la date de l'incorporation dans le sens direct et tantôt d'après ce qu'on appelle «la classe », d'après l'intervalle qui vous en sépare, c'est-à-dire en sens inverse, peut-être parce que la monotonie des journées fait que cette durée se rapproche le plus du temps homogène où, pour la mesure, on peut choisir par convention le sens que l'on veut. Ainsi autant il y a de groupes, autant il y a d'origines des temps différentes. Il n'y en a aucun qui s'impose à tous les groupes.

Mais il en est de même du jour. On pourrait croire que l'alternance des jours et des nuits marque une division fondamentale, un rythme élémentaire du temps qui est le même dans toutes les sociétés. La nuit consacrée au sommeil interrompt, en effet, la vie sociale. C'est la période où l'homme échappe presque entièrement à l'emprise des lois, des coutumes, des représentations collectives, où il est vraiment seul. Cependant, la nuit est-elle une période exceptionnelle à cet égard, et n'y a-t-il que le sommeil physique qui arrête temporairement la marche de ces courants que sont les sociétés ? Si nous lui attribuons cette 
vertu, c'est que nous oublions qu'il n'y a pas une société seulement, mais des groupes, et que la vie de nombre d'entre eux s'interrompt bien avant la nuit et à d'autres moments encore. Disons, si l'on veut, qu'un groupe s'endort lorsqu'il n'y a plus d'hommes associés pour soutenir et dérouler sa pensée, mais qu'il sommeille seulement, qu'il continue d'exister tant que ses membres sont prêts à se rapprocher et à le reconstituer tel qu'il était lorsqu'ils l'ont quitté. Or, il n'y a qu'un groupe dont on puisse dire que sa vie consciente est périodiquement suspendue par le sommeil physique des hommes, c'est la famille, puisqu'en général ce sont les siens dont on prend congé quand on se couche et qu'on voit avant tous au réveil. Mais la conscience du groupe familial s'obscurcit et s'évanouit encore à d'autres moments, quand ses membres s'éloignent, le père et quelquefois la mère à leur travail, l'enfant à l'école, et les périodes d'absence qui, comptées en heures d'horloge, sont plus courtes que la nuit, ne paraissent peut-être pas moins longues à la famille elle-même, car pendant la nuit elle n'a pas conscience du temps ; qu'un homme ait dormi une heure ou dix heures, au réveil il ne sait combien de temps s'est écoulé : une minute, une éternité ? Quant aux autres groupes, c'est, en général, bien avant la nuit que leur vie s'interrompt et bien après qu'elle reprend. Si, d'ailleurs, cette interruption est plus longue, elle n'est pas d'une autre nature que d'autres arrêts qui se produisent dans la vie des mêmes groupes à d'autres moments de la journée. En tout cas, la journée de travail ne s'étend pas de façon ininterrompue sur toute la suite des heures qui séparent le réveil du sommeil ; elle n'atteint pas ces deux limites et elle est coupée d'intervalles qui appartiennent à d'autres groupes. Il en est de même, à plus forte raison, de la journée religieuse ou de la journée mondaine. Si la nuit nous paraît cependant marquer la division essentielle du temps, c'est qu'elle l'est, en effet, pour la famille et qu'il n'est pas de communauté à laquelle nous nous rattachions plus étroitement. Mais tenons-nous-en aux autres groupes, dont la vie tantôt s'arrête et tantôt reprend ; supposons que les intervalles d'arrêt soient aussi vides que la nuit et que la représentation du temps y disparaisse alors aussi complètement. Il serait bien difficile de dire dans ces groupes où commence le jour et où il finit, et en tout cas, il ne commencerait pas au même moment dans tous les groupes.

En fait, cependant, nous l'avons vu, il y a une correspondance assez exacte entre tous ces temps, bien qu'on ne puisse dire qu'ils sont 
adaptés l'un à l'autre par une convention établie entre les groupes. Tous divisent le temps en gros de la même manière parce qu'ils ont tous hérité, à cet égard, d'une même tradition. Cette division traditionnelle de la durée s'accorde d'ailleurs avec le cours de la nature, et il n'y a pas à s'en étonner, puisqu'elle a été établie par des hommes qui observaient le cours des astres et le cours du soleil. Comme la vie de tous les groupes se déroule dans les mêmes conditions astronomiques, ils peuvent tous constater que le rythme du temps social et l'alternance des phénomènes de la nature sont bien adaptés l'un à l'autre. Il n'en est pas moins vrai, que, d'un groupe à l'autre, les divisions du temps qui s'accordent ne sont pas les mêmes et n'ont pas en tout cas le même sens. Tout se passe comme si un même balancier communiquait son mouvement à toutes les parties du corps social. Mais, en réalité, il n'y a pas un calendrier unique, extérieur aux groupes et auquel ils se réfèreraient. Il y a autant de calendriers que de sociétés différentes, puisque les divisions du temps s'expriment tantôt en termes religieux (chaque jour étant consacré à un saint), tantôt en termes d'affaires (jours d'échéance, etc.). Il importe peu qu'ici et là on parle de jours, de mois, d'années. Un groupe ne pourrait se servir du calendrier d'un autre. Ce n'est pas dans le temps religieux que vit le marchand et qu'il y peut trouver des points de repère. S'il en a été autrement à des époques plus ou moins éloignées, si les foires et les marchés se plaçaient à des jours consacrés par la religion, si l'expiration d'une dette de commerce se plaçait à la Saint-Jean, à la Chandeleur, c'est que le groupe économique ne s'était pas encore détaché de la société religieuse.

Mais la question se pose alors de savoir si ces groupes eux-mêmes sont vraiment séparés. On pourrait concevoir, en effet, non seulement qu'ils se fassent de nombreux emprunts, mais que leurs vies se rapprochent et se fondent bien souvent, que ces lignes d'évolution se croisent sans cesse. Si plusieurs courants de pensée collective peuvent ainsi, au moins à intervalles, se mêler, échanger leur substance et couler dans un même lit, comment parler de temps multiples ? N'est-ce pas dans un même temps qu'ils fixent la place d'une partie au moins de leurs souvenirs ? Si nous suivons la vie d'un groupe tel que l'Église pendant une période de son évolution, nous verrons que sa pensée a reflété la vie d'autres sociétés contemporaines avec lesquelles elle s'est trouvée en contact. Quand Sainte-Beuve écrit Port-Royal, il entre d'autant plus profondément dans ce mouvement religieux unique en son genre, il en 
atteint d'autant mieux les secrets ressorts et l'originalité interne, qu'il fait entrer dans son tableau un plus grand nombre de faits et de personnages empruntés à d'autres milieux, mais qui marquent autant de points de contact entre le siècle et les préoccupations de ces solitaires. Il n'est guère d'événement religieux qui n'ait une face tournée vers la vie du dehors et n'ait son retentissement dans les groupes laïcs. Qu'on tienne note des propos échangés dans une réunion de famille ou dans un salon ; il y sera question de ce qui se passe dans d'autres familles, dans d'autres milieux, comme si le groupe des artistes, le groupe des politiciens pénétraient à l'intérieur de ces assemblées si différentes, ou les entraînaient dans leur mouvement. Quand on dit d'une société (d'une famille, d'un milieu mondain), qu'elle est vieux jeu ou qu'elle est dans le train, n'est-ce pas à des pénétrations ou contaminations de ce genre qu'on pense ? Puisque tout fait notable, en quelque région du corps social qu'il ait pris naissance, peut être pris comme point de repère par n'importe quel groupe, pour déterminer les époques de sa durée, n'est-ce pas la preuve que les limites qu'on trace entre les divers courants collectifs sont arbitraires, et qu'ils se touchent en trop de points de leur parcours pour qu'il y ait heu de les séparer?

On dit qu'un même événement peut affecter à la fois plusieurs consciences collectives distinctes; on en conclut qu'à ce moment ces consciences se rapprochent et s'unissent dans une représentation commune. Mais est-ce bien un même événement si chacune de ces pensées se le représente à sa manière et le traduit dans son langage ? Il s'agit de groupes qui sont l'un et l'autre dans l'espace. L'événement aussi se produit dans l'espace, et il se peut que l'un et l'autre groupe le perçoivent. Mais ce qui importe, c'est la façon dont ils l'interprètent, le sens qu'ils lui donnent. Pour qu'ils lui prêtent la même signification, il faut qu'au préalable les deux consciences soient confondues. Or, par hypothèse elles sont distinctes. De fait, il n'est guère concevable que deux pensées pénètrent ainsi l'une dans l'autre. Sans doute, il arrive que deux groupes se fondent, mais alors il naît une conscience nouvelle, dont ni l'étendue ni le contenu ne sont les mêmes qu'auparavant. Ou bien cette fusion n'est qu'apparente si ensuite les deux groupes se séparent et se retrouvent pour l'essentiel tels qu'ils étaient autrefois. Un peuple qui en conquiert un autre peut se l'assimiler ; mais alors, lui-même devient un autre peuple, ou tout au moins entre dans une nouvelle phase de son existence. S'il ne se l'assimile pas, chacun des 
deux peuples garde sa conscience nationale propre et réagit de façon différente en présence des mêmes événements. Mais il en est de même dans un même pays de la société religieuse et de la société politique. Que l'État se subordonne l'Église, qu'il la remplisse de son esprit, l'Église devient un organe de l'État et perd sa nature de société religieuse ; le courant de pensée religieux se réduit à un mince filet dans> partie de l'Église qui ne se résigne pas à disparaître. Quand l'Église et l'État sont séparés, un même événement, la Réforme, par exemple, donnera lieu dans les âmes religieuses et dans l'esprit des chefs politiques à des représentations différentes qui se relieront tout naturellement aux pensées et traditions des deux groupes, mais qui ne se confondront pas.

De même, si la publication des Lettres provinciales marque une date dans l'histoire de la littérature et dans la vie de Port-Royal, ne nous figurons pas que cette année-là, le courant de la pensée littéraire et le courant religieux janséniste se sont confondus. Nous savons bien que Pascal n'a pas réconcilié $M$. de Sacy avec Montaigne, que les jansénistes n'ont pas cessé de condamner la concupiscence de l'esprit, que pour eux Pascal n'était qu'un instrument de Dieu, et qu'ils attachaient peut-être plus d'importance au miracle de la Sainte-Épine, dont il avait été favorisé dans sa famine, qu'à son activité d'écrivain. Lorsque Sainte-Beuve nous trace le portrait de ceux qui sont entrés à PortRoyal, nous saisissons sur le vif le dédoublement de leur personne : ce sont bien les mêmes hommes ; mais sont-ce les mêmes figures, celles dont le monde a gardé le souvenir et celles qui se sont imposées à la mémoire des jansénistes, tout le brillant de l'esprit, du talent, s'étant éteint, la conversion marquant une fin dans une société et dans l'autre un commencement, comme s'il y avait là deux dates qui n'ont point leur place dans le même temps ? Lorsqu'il s'agit comme ici d'un événement, d'une démarche morale, la question, il est vrai, se complique un peu. Il est concevable que, par exemple, le groupe religieux et telle famille en soient affectés de la même manière parce que la famille, elle-même, est très religieuse. Lorsque Mme Périer raconte la vie de son frère, elle en parle comme d'un saint avec un accent très janséniste. Mais de même dans une famine qui se passionne pour la politique, les discussions qui s'y rapportent mettent la famille en contact avec les milieux dont ces débats sont l'objet exclusif Regardons-y cependant d'un peu plus près. Il y a toujours au moins une nuance ou 
l'absence d'une nuance qui nous découvre si la religion ou la politique a fait passer à l'arrière-plan toutes les considérations de parenté, auquel cas, bien entendu, nous ne sommes plus dans la famille. Il y a eu des moments où la chambre de Pascal se transformait en une cellule ou une chapelle, et où le salon de Mme Rolland ne se distinguait plus d'un club ou d'un Conseil des ministres girondins. Au contraire, dans d'autres cas, la pensée familiale s'empare des images et événements de la religion et de la politique pour alimenter sa vie propre, qu'on s'enorgueillisse de l'éclat qui rejaillit sur la famille de ce qu'un de ses membres s'est illustré dans l'un ou l'autre de ces domaines, que ses membres se sentent comme parents plus rapprochés ou déchirés au contraire parce que leurs croyances et convictions à cet égard les unissent ou les séparent. Mais cela n'est possible que si ces éléments de pensée qui se rapportent en apparence, pour la famille, à des objets et personnes qui lui sont extérieurs, se transposent en représentations familiales, c'est-à-dire conservent bien la forme apparente, politique ou religieuse, mais aient comme substance les réactions de la parenté, les intérêts et préférences de la maison, des frères, des ascendants. Que de telles transpositions soient possibles, c'est ce qui résulte de ce que le plus souvent on pratique telle religion et l'on suit telle opinion politique parce que ce sont depuis longtemps celles de la famille. « Mon Dieu et mon Roi », dit le paysan, mais il faut entendre " Mon foyer, mes parents ». Que d'oppositions, de croyances et convictions qui ne sont aussi qu'un antagonisme déguisé de frère à frère, d'enfants à parents ! Ce qui n'empêche qu'à certains moments toutes préoccupations de famille disparaissent, et les parents sont oubliés ; c'est alors qu'on est pris vraiment dans les groupes religieux et politiques, comme on le serait aussi dans les groupes qui s'occupent de sciences, arts et affaires ; mais alors, il ne faut pas que s'entretenant de ces choses avec les siens, on oublie ces groupes pour penser aux siens.

Si les divers courants de pensée collective ne pénètrent réellement jamais l'un dans l'autre, et ne peuvent être mis et maintenus en contact, il est bien difficile de dire si le temps s'écoule plus vite pour l'un que pour l'autre. Comment connaîtrait-on la vitesse du temps puisqu'il n'y a pas de commune mesure, et que nous ne concevons aucun moyen de mesurer la vitesse de l'une par rapport à celle de l'autre ? On a beau dire que dans certains milieux la vie s'écoule, les pensées et sentiments se succèdent suivant un rythme plus rapide qu'ail- 
leurs. Définirons-nous la vitesse du temps d'après le nombre d'événements qu'il renferme ? Mais nous l'avons dit, le temps est tout autre chose qu'une série successive de faits, ou qu'une somme de différences. On est victime d'une illusion lorsqu'on se figure qu'une plus grande quantité d'événements ou de différences signifie la même chose qu'un temps plus long. C'est oublier que les événements divisent le temps, mais ne le remplissent pas. Ceux qui multiplient leurs occupations et leurs distractions finissent par perdre la notion du temps réel, et peut-être par faire s'évanouir la substance même du temps, qui, découpé en tant de parties, ne peut plus s'étendre et se dilater, et n'offre plus aucune consistance. Comme la faculté de changer, pour un groupe humain, est limitée, il faut bien qu'à mesure que les changements se multiplient, dans une même durée de vingt-quatre heures, chacun d'eux devienne moins important. De fait, l'activité de groupes comme les bourses de titres, les sociétés industrielles et commerciales, où se traitent en peu de temps une quantité d'affaires, est presque toujours du genre mécanique. Ce sont les mêmes calculs, les mêmes types de combinaisons qui défilent dans la pensée de leurs membres. Il faudra attendre plusieurs années, quelquefois plusieurs décennies, pour que de l'accumulation de toutes ces paroles et de tous ces gestes, il sorte un changement important, qui modifie de façon durable la mémoire de ces milieux, c'est-à-dire l'image qu'ils gardent de leur passé. À travers cette agitation plus qu'à demi automatique, le groupe retrouve un temps assez uniforme, et qui ne s'écoule, en somme, pas plus rapidement que celui du pêcheur à la ligne. On répète aussi qu'il y a des peuples retardataires, dont l'évolution s'est accomplie très lentement, et, dans un même pays, c'est un lieu commun d'insister sur le rythme rapide de l'existence dans les grandes villes par contraste avec les petits bourgs, ou dans les régions industrielles par contraste avec la campagne.

N'oublions pas, cependant, que les groupes que l'on compare n'ont ni la même nature, ni le même genre d'occupation. Mais, de ce que, dans l'intervalle d'une journée, les habitants d'un village ont moins d'occasions de changer la direction de leur activité ou de leur pensée, s'ensuit-il que, pour eux, le temps s'écoule plus lentement que dans les villes ? C'est l'habitant de la ville qui a cette idée, mais pourquoi ? Parce qu'il se représente le village comme une ville dont l'activité s'est ralentie, qui s'est petit à petit paralysée et endormie. Mais un village 
est un village et il faut le comparer à lui-même et non a un groupe d'une autre nature. Or, à la campagne, le temps se divise suivant un ordre d'occupations qui se règlent elles-mêmes sur le cours de la nature animale ou végétale. Il faut attendre que le blé soit sorti de la terre, que les bêtes aient fait leurs oeufs ou leurs petits, que les pis des vaches se soient remplis. Il n'y a pas de mécanisme qui puisse précipiter ces opérations. Le temps est bien ce qu'il doit être dans un tel groupe, et chez de tels hommes dont la pensée a pris une allure conforme à leurs besoins et à leurs traditions. Sans doute il y a des périodes où l'on se presse et des jours où l'on se repose, mais ce sont des irrégularités qui portent sur le contenu du temps et n'altèrent point son cours. Qu'on s'absorbe dans sa tâche, dans une causerie, dans une rêverie, une réflexion, un souvenir, qu'on regarde passer les gens, ou qu'on joue aux cartes, du moment que ce sont là des manières d'être et des activités habituelles, réglées par la coutume, et que chacune a la place et la durée qui lui convient, le temps est bien ce qu'il a toujours été, ni trop rapide ni trop lent. Inversement des paysans transportés dans une ville s'étonneront de ce que le rythme de la vie se précipite, et penseront qu'une journée étant plus remplie doit condenser aussi plus de temps. C'est qu'ils se représentent la ville comme un village saisi d'une fièvre d'activité, où les hommes sont surexcités, où pensées et gestes sont entraînés dans un mouvement vertigineux. Mais la ville est la ville, c'est-à-dire un milieu où le mécanisme s'est introduit, non seulement dans les travaux productifs, mais règle aussi les déplacements, les distractions et le jeu de l'esprit. Le temps est divisé comme il doit l'être, il est ce qu'il doit être, ni trop rapide, ni trop lent, puisqu'il est conforme aux besoins de la vie urbaine. Les pensées qui le remplissent sont plus nombreuses, mais aussi plus courtes : elles ne peuvent pousser de profondes racines dans les esprits. C'est qu'une pensée ne prend consistance que lorsqu'elle s'étend sur une durée suffisante. Mais comment comparer le nombre des états de conscience qui se succèdent pour mesurer la rapidité du temps dans les deux groupes, puisqu'il ne s'agit pas de pensées et représentations du même genre ? En réalité, on ne peut dire que le temps s'écoule plus vite ou plus lentement dans une société que dans une autre ; la notion de rapidité, appliquée au cours du temps, n'offre pas une situation définie. Par contre, c'est un fait remarquable que la pensée, lorsqu'on se souvient, peut parcourir en quelques instants des intervalles de temps plus ou moins grands, et remonter le cours de la durée avec une rapidité 
qui varie non seulement d'un groupe à l'autre, mais encore à l'intérieur d'un groupe, d'un individu à l'autre, et même, pour un individu demeurant dans le même groupe, d'un moment à l'autre. On s'étonne quelquefois, lorsqu'on cherche un souvenir très éloigné, de la légèreté avec laquelle l'esprit saute par-dessus de vastes périodes et, comme s'il avait mis des bottes de sept lieues, entrevoit à peine au passage les représentations du passé qui, apparemment, remplissent l'intervalle.

Mais pourquoi imaginer que tous ces anciens souvenirs sont là, rangés, suivant l'ordre même où ils se sont succédé comme s'ils nous attendaient ? Si, pour remonter dans le passé, il fallait se guider sur ces images toutes différentes l'une de l'autre, chacune correspondant à un événement qui n'a eu heu qu'une fois, alors l'esprit ne passerait point au-dessus d'eux à vastes enjambées, il ne se bornerait même pas à les effleurer, mais ils défileraient un à un sous son regard. En réalité, l'esprit ne passe pas en revue toutes ces images, dont rien n'indique d'ailleurs qu'elles subsistent. C'est dans le temps, dans un temps qui est celui d'un groupe donné, qu'il cherche à retrouver ou reconstituer le souvenir et c'est sur le temps qu'il prend son appui. Le temps peut et peut seul jouer ce rôle dans la mesure où nous nous le représentons comme un milieu continu qui n'a pas changé, qui est resté tel aujourd'hui qu'hier, en sorte que nous pouvons retrouver hier dans aujourd'hui. Que le temps puisse demeurer en quelque sorte immobile pendant une période assez étendue, cela résulte de ce qu'il sert de cadre commun à la pensée d'un groupe qui, lui-même, pendant cette période ne change pas de nature, qui conserve à peu près la même structure, et tourne son attention vers les mêmes objets. Tant que ma pensée peut remonter dans un temps de ce genre, le redescendre, en explorer les diverses parties d'un mouvement continu, sans se heurter à un obstacle ou à une barrière qui l'empêche de voir au-delà, elle se meut dans un milieu dont tous les éléments se tiennent. Il suffit qu'elle se déplace dans ce milieu pour qu'elle en retrouve tous les éléments. Bien entendu ce temps ne se confond point avec les événements qui s'y sont succédé, mais il ne se réduit pas non plus, nous l'avons montré, à un cadre homogène et entièrement vide. On y trouve inscrite ou indiquée la trace des événements ou des figures d'autrefois dans la mesure où ils répondaient et répondent encore à un intérêt ou à une préoccupation du groupe. Quand nous disons que l'individu s'aide de la mémoire du groupe, il faut bien entendre que cette aide n'implique pas la présence 
actuelle d'un ou plusieurs de ses membres. En effet, je continue à subir l'influence d'une société, alors même que je m'en suis éloigné ; il suffit que je porte avec moi dans mon esprit tout ce qui est en mesure de me placer au point de vue de ses membres, de me replonger dans leur milieu et dans leur temps propre, et de me sentir au cœur du groupe. Ceci demande, il est vrai, quelque explication.

je me revois, en pensée, à côté d'un camarade d'école avec qui j'étais très lié, engagés tous deux dans une conversation psychologique ; nous analysions et décrivions les caractères de nos maîtres, de nos amis. Lui et moi faisions partie du groupe de nos camarades, mais, dans ce groupe, nos relations personnelles, et d'ailleurs antérieures à notre entrée à l'école, avaient créé entre nous une communauté plus étroite. Il y a de longues années que je ne l'ai vu, mais notre groupe subsiste en pensée au moins, car si nous nous rencontrions demain, nous aurions l'un vis-à-vis de l'autre la même attitude que quand nous nous sommes quittés. Seulement, il est mort il y a quelques mois. Alors notre groupe est dissous. je ne le rencontrerai plus. je ne peux plus l'évoquer comme une personne actuellement vivante. Quand je nous vois maintenant engages autrefois dans une conversation, comment prétendre que pour évoquer ces souvenirs, je m'appuie sur la mémoire de notre groupe, puisque notre groupe n'existe plus ? Mais le groupe n'est pas seulement, ni même surtout, un assemblage d'individus définis et sa réalité ne s'épuise pas dans quelques figures que nous pouvons énumérer, et à partir desquelles nous le reconstruirions. Bien au contraire, ce qui le constitue essentiellement, c'est un intérêt, un ordre d'idées et de préoccupations, qui, sans doute, se particularisent et reflètent dans une certaine mesure les personnalités de ses membres, mais qui sont cependant assez générales et même impersonnelles pour conserver leur sens et leur portée pour moi, alors même que ces personnalités se transformeraient et que d'autres, semblables, il est vrai, mais différentes, leur seraient substituées. C'est là ce qui représente l'élément stable et permanent du groupe, et, loin de le retrouver à partir de ses membres, c'est à partir de cet élément que je reconstruis les figures de ceux-ci. Si donc je pense à mon ami, c'est que je me replace dans un courant d'idées qui nous ont été communes, et qui subsiste pour moi, alors même que mon ami n'est plus là, ou ne peut plus à l'avenir me rencontrer, pourvu que se conservent autour de moi les conditions qui me permettent de m'y replacer. Or, elles se 
conservent, car de telles préoccupations n'étaient pas étrangères à nos amis communs, et j'ai rencontré, je rencontre encore des personnes qui ressemblent à mon ami, au moins sous ce rapport, chez qui je retrouve le même caractère et les mêmes pensées, comme si elles avaient été des membres virtuels du même groupe.

Supposons que les relations entre deux ou plusieurs personnes soient telles que cet élément de pensée commune impersonnelle fasse défaut. Deux êtres s'aiment d'une passion étroitement égoïste, la pensée de chacun est tout entière remplie par l'autre. Ils peuvent dire : je l'aime parce que c'est lui ou parce que c'est elle. Ici point de substitution possible. Mais aussi, la passion disparue, il ne subsistera rien du lien qui les unissait, et alors ou bien ils s'oublieront ou bien ils ne garderont l'un de l'autre qu'un souvenir pâle et décoloré. Sur quoi s'appuieraient-ils, en effet, pour que chacun se rappelle l'autre tel qu'il le voyait ? Quelquefois cependant, si le souvenir subsiste malgré l'éloignement, malgré la mort, c'est, qu'outre l'attachement personnel, il y avait une pensée commune, le sentiment de la fuite du temps, la vue des objets environnants, la nature, quelque sujet de méditation : c'est l'élément stable qui transformait l'union de deux êtres à base simplement affective en une société, et c'est la pensée subsistante du groupe qui évoque le rapprochement passé, et qui sauve de l'oubli l'image de la personne. Auguste Comte aurait-il pu évoquer Clotilde de Vaux et la voir presque avec les yeux du corps, si leur amour n'avait point pris le sens d'une union spirituelle, et s'il ne l'avait point replacée dans la religion de l'humanité ? C'est ainsi qu'on se rappelle ses parents sans doute parce qu'on les aime, mais surtout parce qu'ils sont vos parents. Deux amis ne s'oublient pas, parce que l'amitié suppose un accord des pensées et quelques préoccupations communes.

En réalité, nos relations avec quelques personnes s'incorporent à des ensembles plus larges, dont nous ne nous représentons pas sous forme concrète les autres membres. Ces ensembles tendent à dépasser les figures que nous connaissons, et presque à se dépersonnaliser. Or, ce qui est personnel est aussi plus stable. Le temps où a vécu le groupe est un milieu à demi dépersonnalisé, où nous pouvons assigner la place de plus d'un événement passé, parce que chacun d'eux a une signification par rapport à l'ensemble. C'est cette signification que nous retrouvons dans l'ensemble et celui-ci se conserve parce que sa 
réalité ne se confond pas avec les figures particulières et passagères qui le traversent.

Cette permanence du temps social est d'ailleurs toute relative. En fait, si notre emprise sur le passé, dans les directions diverses où s'engage la pensée de ces groupes, s'étend assez loin, elle n'est pas illimitée, et ne dépasse jamais une ligne qui se déplace à mesure que les sociétés dont nous sommes membres entrent dans une nouvelle période de leur existence. Tout se passe en apparence comme si la mémoire avait besoin de s'alléger, quand grossit le flot des événements qu'elle doit retenir. Remarquons-le d'ailleurs, ce n'est pas le nombre des souvenirs qui importe ici. Tant que le groupe ne change pas sensiblement, le temps que sa mémoire embrasse peut s'allonger : c'est toujours un milieu continu, qui nous reste accessible dans toute son étendue. C'est lorsqu'il se transforme qu'un temps nouveau commence pour lui, et que son attention se détourne progressivement de ce qu'il a été, et de ce qu'il n'est plus maintenant. Mais le temps ancien peut subsister à côté du temps nouveau, et même en lui, pour ceux de ses membres qu'une telle transformation a le moins touchés, comme si le groupe ancien refusait de se laisser entièrement résorber dans le groupe nouveau qui est sorti de sa substance. Si la mémoire atteint alors des régions du passé inégalement éloignées suivant les parties du corps social que l'on envisage, ce n'est pas parce que les uns ont plus de souvenirs que les autres : mais les deux parties du groupe organisent leur pensée autour de centres d'intérêts qui ne sont plus tout à fait les mêmes.

Sans sortir de la famille, la mémoire du père et de la mère les transporte dans le temps qui suivit leur mariage ; elle explore une région du passé que les enfants ne connaissent que par ouï-dire ; ceux-ci n'ont pas de souvenir d'un temps où ils ne s'étaient pas encore éveillés à la conscience au milieu de leurs parents. La mémoire du groupe familial se réduit-elle alors à un faisceau de séries de souvenirs individuels, semblables pour toute la partie du temps où elles correspondent aux mêmes circonstances, mais qui, lorsqu'on remonte le cours de la durée, s'interrompent plus ou moins haut ? Ainsi dans une famille, autant de mémoires, autant de vues sur un même groupe qu'il y a de membres dans la famille, puisqu'elles s'étendent sur des temps iné- 
gaux ? Non, mais plutôt reconnaissons dans la vie de ce groupe des transformations caractéristiques.

Jusqu'au moment où les enfants sont nés et sont devenus capables de se souvenir, et depuis le mariage, il a pu s'écouler peu de temps. Mais cette année ou ces quelques années sont remplies d'événements, alors même qu'en apparence il ne s'y passe rien. C'est alors que se découvrent, non seulement les caractères personnels des deux époux, mais tout ce qu'ils tiennent de leurs parents, des milieux où ils ont vécu jusque-là ; pour qu'un groupe nouveau s'édifie sur ces éléments, il faut toute une série d'efforts en commun à travers bien des étonnements, des résistances, des conflits, des sacrifices, mais aussi bien des accords spontanés et des rencontres, des assentiments, des encouragements, des découvertes faites ensemble dans le monde de la nature et de la société. C'est le temps consacré à établir les fondements de l'édifice, temps plus pittoresque et plus mouvementé que les longs intervalles où s'achèvera la maison; il y a sur le chantier une effervescence, un élan unanime, d'abord parce que c'est un commencement. Plus tard, on sera obligé de régler son travail sur ce qui a déjà été réalisé, dont on a la responsabilité en même temps que l'orgueil ; de se mettre à l'alignement des édifices voisins ; de tenir compte des exigences et des préférences de ceux qui habiteront la maison, qu'on ne prévoit pas toujours; d'où bien des contretemps, du temps perdu, du travail à défaire et à refaire. Mais aussi, on sera exposé à s'arrêter au milieu du travail pour une raison ou l'autre. Il y a des maisons inachevées, des travaux qui attendent longtemps qu'on les reprenne. Pendent opera interrupta. Il y a aussi l'ennui de revenir travailler au même endroit jour après jour. Dans l'activité même de ceux qui terminent un bâtiment, il y a souvent plus d'inquiétude que d'allégresse. Un chantier de démolition évoque toujours un peu la nature, et les ouvriers qui creusent les fondations ressemblent à des pionniers. Comment la période où l'on pose les bases d'un groupe nouveau ne serait-elle pas remplie des pensées les plus intenses et destinées le plus à durer ? Dans plus d'une société survit ainsi l'esprit des fondateurs, si court qu'ait pu être le temps consacré à la fondation.

Dans beaucoup de cas, la venue des enfants n'élargit pas seulement la famille, elle modifie sa pensée et la direction de son intérêt. L'enfant est toujours un intrus, en ce sens qu'on sait bien qu'il ne s'adaptera 
pas à la famille déjà constituée, mais que les parents, et même les enfants déjà nés, devront se plier, sinon aux exigences du nouveau venu, du moins aux changements qui résultent de son intégration dans le groupe. jusqu'ici, le couple sans enfants a pu penser qu'il se suffirait à lui-même ; peut-être s'est-il à peu près suffi en apparence, alors qu'il s'ouvrait à beaucoup d'influences du dehors : lectures, théâtre, relations, voyages, occupations professionnelles de l'homme et peut-être de la femme, le tout mis en commun, et dans ce passage à travers beaucoup de milieux, le ménage réagissant à sa manière et prenant de mieux en mieux conscience de son unité. Il est pris entre deux dangers : se trop resserrer et replier sur lui-même, ne plus garder avec les groupes extérieurs même le contact que permet la lecture, ce qui le condamne à dépérir, car il ne peut vivre que de substance sociale, et c'est pourquoi il aspire toujours à sortir du cercle de ses membres et à se répandre. Mais l'autre risque, c'est de se répandre trop, de se laisser absorber par un groupe extérieur au ménage ou par quelque préoccupation qui lui soit trop excentrique. Il en résulte parfois, du moins, au début, une alternance de périodes où le ménage, cherchant en quelque sorte sa place dans la société extérieure, tantôt se laisse prendre par elle et tantôt se tient à l'écart ; contrastes qui ressortent assez vivement pour que cette phase de sa vie se détache elle-même des suivantes et reste gravée dans sa mémoire. Plus tard, il a trouvé sa place : il a ses relations, ses intérêts, son rang; ses liaisons avec les autres groupes sont à peu près stabilisées; ses préoccupations essentielles ont pris une forme plus arrêtée. A plus forte raison, quand un ménage a des enfants, ses rapports avec le milieu social qui l'environne se multiplient et se définissent. Quand un groupe comprend plus de membres, surtout quand ceux-ci sont d'âge différent, il entre en contact avec la société par plus de parties de lui-même. Il s'incorpore plus étroitement au milieu qui comprend les autres familles, se pénètre de son esprit, se plie à ses règles. On pourrait penser qu'une famille plus large se suffit davantage à elle-même et constitue un milieu plus fermé. Ce n'est pas entièrement exact. Certes les parents ont maintenant une préoccupation commune nouvelle et singulièrement forte. Mais le groupe familial, plus étendu, a plus de peine à s'isoler matériellement ; il offre une surface plus large aux regards des autres, une prise plus grande à l'opinion. La famille est faite d'un ensemble de relations internes plus nombreuses et plus complexes, plus impersonnelles aussi, puisqu'elle réalise à sa manière un type d'organisation domestique qui existe en 
dehors d'elle et qui tend à la dépasser. A cette transformation du groupe correspond un remaniement profond de sa pensée. C'est comme un nouveau point de départ. Pour les enfants, c'est toute la vie de la famille, du moins celle dont ils gardent quelque souvenir. La mémoire des parents remonte plus haut, sans doute parce que le groupe qu'ils formaient autrefois ne s'est pas entièrement résorbé dans la famille élargie. Il a continué à exister, mais d'une vie discontinue et comme amortie. On s'en aperçoit lorsque les enfants s'éloignent. On éprouve alors une impression d'irréalité comme lorsque deux amis se retrouvent après bien longtemps, peuvent bien évoquer le passé commun, mais n'ont rien de plus à se dire. On est comme à l'extrémité d'un chemin qui se perd, ou comme deux partenaires qui ont oublié les règles du jeu. Ainsi, lorsqu'une société s'est trouvée soumise à un remaniement profond, il semble que la mémoire atteigne par deux voies différentes les souvenirs correspondant à ces deux périodes successives, et ne remontent pas de l'une à l'autre d'une manière continue. Il y a en réalité deux temps où se conservent deux cadres de pensée, et c'est tantôt dans l'un, tantôt dans l'autre qu'il faut se replacer pour retrouver les souvenirs dans chacun des cadres où ils sont localisés. Pour retrouver une ville ancienne dans le dédale des rues nouvelles qui l'ont peu à peu encerclée et bouleversée, des maisons et monuments qui tantôt ont découvert et effacé les anciens quartiers et tantôt trouvé leur emplacement sur le prolongement et dans l'intervalle des constructions d'autrefois, on ne remonte pas du présent au passé en suivant en sens inverse, et de façon continue, la série des travaux, démolitions, tracés de voies, etc., qui ont modifie progressivement l'aspect de cette cité. Mais pour retrouver les voies et monuments anciens, conservés d'ailleurs ou disparus, on se guide sur le plan général de la ville ancienne, on s'y transporte en pensée, ce qui est toujours possible à ceux qui y ont vécu avant qu'on ait élargi et rebâti les vieux quartiers, et pour qui ces pans de murs restés debout, ces façades d'un autre siècle, ces tronçons de rues gardent leur signification d'autrefois. Dans la ville moderne elle-même, on retrouve les particularités de la ville ancienne, parce qu'on n'a d'yeux et de pensée que pour celle-ci. Ainsi, lorsque dans une société qui s'est transformée subsistent des vestiges de ce qu'elle était primitivement, ceux qui l'ont connue en son premier état peuvent aussi fixer leur attention sur ces traits anciens qui leur ouvrent l'accès d'un autre temps et d'un autre passé. Il n'est guère de société où nous ayons vécu quelque temps qui ne subsiste, du 
moins qui n'ait laissé quelque trace d'elle-même dans des groupes plus récents où nous sommes plongés ; la subsistance de ces traces suffit à expliquer la permanence et la continuité du temps propre à cette société ancienne et qu'il nous soit possible à tout moment d'y repénétrer par la pensée.

Tous ces temps, subsistant encore même lorsqu'ils correspondent aux états et comme aux formes successives d'une société qui a profondément évolué, sont impénétrables l'un à l'autre. Ils subsistent d'ailleurs, l'un à côté de l'autre. En effet, les groupes dont les pensées sont distinctes sont étendus matériellement dans l'espace, et les membres dont ils se composent entrent à la fois ou successivement dans plusieurs d'entre eux. Il n'y a pas un temps universel et unique, mais la société se décompose en une multiplicité de groupes, dont chacun a sa durée propre. Ce qui distingue ces temps collectifs, ce n'est pas que les uns s'écoulent plus vite que les autres. On ne peut même pas dire que ces temps s'écoulent, puisque chaque conscience collective peut se souvenir, et que la subsistance du temps paraît bien être une condition de la mémoire. Les événements se succèdent dans le temps, mais le temps lui-même est un cadre immobile. Seulement, les temps sont plus ou moins vastes, ils permettent à la mémoire de remonter plus ou moins loin dans ce qu'on est convenu d'appeler le passé. Plaçons-nous maintenant au point de vue des individus. Chacun est membre de plusieurs groupes, il participe à plusieurs pensées sociales, son regard plonge successivement dans plusieurs temps collectifs. C'est là déjà un élément de différenciation individuelle : dans une même période, en une région de l'espace, ce n'est pas entre les mêmes courants collectifs que se partagent les consciences des divers hommes. Mais, en outre, leurs pensées remontent plus ou moins loin, plus ou moins vite dans le passé ou dans le temps de chaque groupe. C'est en ce sens que les consciences concentrent en un même intervalle des durées plus ou moins étendues; disons qu'en un même intervalle de durée sociale vécue, elles font tenir une étendue plus ou moins grande de temps représenté. Il y a bien entendu à cet égard de grandes différences entre elles.

Tout autre est l'interprétation des psychologues qui croient qu'il y a autant de durées différentes irréductibles l'une à l'autre que de consciences individuelles, parce que chacune d'elles est comme un flot de 
pensée qui s'écoule avec son mouvement propre. Mais d'abord, le temps ne s'écoule pas : il dure, il subsiste et il le faut bien, sinon comment la mémoire pourrait-elle remonter le cours du temps ? De plus, chacun de ces courants ne se présente pas comme une série unique et continue d'états successifs se déroulant plus ou moins vite, sinon comment, de leur comparaison, pourrait-on tirer la représentation d'un temps commun à plusieurs consciences ? En réalité, si, en rapprochant plusieurs consciences individuelles, on peut replacer leur pensée ou leurs événements dans un ou plusieurs temps communs, c'est que la durée intérieure se décompose en plusieurs courants qui ont leur source dans les groupes eux-mêmes. La conscience individuelle n'est que le lieu de passage de ces courants, le point de rencontre des temps collectifs.

Il est curieux que cette conception n'ait guère été envisagée jusqu'à présent par les philosophes qui ont étudié le temps. Cela tient à ce qu'on s'est toujours représenté les consciences comme isolées l'une de l'autre, et chacune enfermée en elle-même. L'expression stream of thought, ou encore "flux ou courant psychologique " qu'on trouve dans les écrits de William James et de Henri Bergson, traduit, à l'aide d'une image exacte, le sentiment dont chacun de nous peut faire l'expérience lorsqu'il assiste en spectateur au déroulement de sa vie psychique. Tout paraît, en effet, se passer comme si, à l'intérieur de chacun de nous, nos états de conscience se succédaient comme les tranches d'un courant continu, comme des vagues qui se poussent l'une l'autre. A la réflexion cependant, on s'aperçoit qu'il en est ainsi d'une pensée qui va sans cesse de l'avant, qui passe sans cesse d'une perception à une perception, d'un état affectif à un autre, mais que le propre de la mémoire est, au contraire, qu'elle nous oblige à nous arrêter, à nous détourner momentanément de ce flux et, sinon à remonter le courant, du moins, a nous engager dans une direction de traverse, comme si, le long de cette série continue, se présentaient une quantité de points qui amorcent des bifurcations. Certes, la pensée est encore active dans la mémoire ; elle se déplace ; elle est en mouvement. Mais ce qui est digne de remarque, c'est qu'alors, et alors seulement, on peut dire qu'elle se déplace et se meut dans le temps. Comment, sans la mémoire, et en dehors des moments où l'on se souvient, aurait-on conscience d'être dans le temps et de se transporter à travers la durée ? Lorsqu'on s'absorbe dans ses impressions, lorsqu'on les suit à mesure 
qu'elles apparaissent puis disparaissent, on se confond sans doute avec un moment de la durée, puis avec un autre ; mais comment se représenterait-on le temps lui-même, c'est-à-dire le cadre temporel qui embrasse à la fois ce moment et beaucoup d'autres ? On peut être dans le temps, dans le présent qui est une partie du temps, et cependant ne pas être capable de penser dans le temps, de se transporter par la pensée dans le passé proche ou lointain. En d'autres termes, du courant des impressions, il faut distinguer les courants de la pensée proprement dite ou de la mémoire; les premiers sont étroitement liés à notre corps ; ils ne nous font point sortir de nous, mais ils ne nous ouvrent aucune perspective sur le passé ; les seconds ont leur source et la plus grande partie de leurs cours dans la pensée des groupes divers auxquels nous nous rattachons.

Si nous mettons au premier plan les groupes et leurs représentations, si nous concevons la pensée individuelle comme une série de points de vue successifs sur les pensées de ces groupes, alors nous comprendrons qu'elles puissent remonter dans le passé, et remonter plus ou moins loin, suivant l'étendue des perspectives que lui offre chacun de ces points de vue sur le passé tel qu'il est représenté dans les consciences collectives auxquelles ces pensées participent. La condition nécessaire pour qu'il en soit ainsi, c'est que, dans chacune de ces consciences, le temps passé, une certaine image du temps subsiste et s'immobilise, que le temps dure au moins dans certaines limites variables suivant les groupes. C'est là le grand paradoxe. Mais à la réflexion comment en pourrait-il être autrement ? Comment une société, quelle qu'elle soit, pourrait-elle exister, subsister, prendre conscience d'elle-même, si elle n'embrassait point d'un regard un ensemble d'événements présents et passés, si elle n'avait pas la faculté de remonter le cours du temps, et de repasser sans cesse sur les traces qu'elle a laissées d'elle-même ? Sociétés religieuses, politiques, économiques, familles, groupes d'amis, de relations, et même réunions éphémères dans un salon, dans une salle de spectacle, dans la rue, toutes immobilisent le temps à leur manière, ou imposent à leurs membres l'illusion que pendant une certaine durée, tout au moins, dans un monde qui change sans cesse, certaines zones ont acquis une stabilité et un équilibre relatifs, et que rien d'essentiel ne s'y est transformé pendant une période plus ou moins longue. 
Certes, les limites jusqu'où l'on remonte ainsi dans le passé sont variables suivant les groupes, et c'est ce qui explique que les pensées individuelles, suivant les moments, c'est-à-dire suivant le degré de leur participation à telle ou telle pensée collective, atteignent des souvenirs plus ou moins éloignés. Au-delà de cette frange mouvante du temps, ou plus exactement des temps collectifs, il n'y a plus rien, car le temps des philosophes n'est qu'une forme vide. Le temps n'est réel que dans la mesure où il a un contenu, c'est-à-dire où il offre une matière d'événements à la pensée. Il est limité et relatif, mais il a une pleine réalité. Il est assez large d'ailleurs pour offrir aux consciences individuelles un cadre suffisamment étoffé pour qu'elles y puissent disposer et retrouver leurs souvenirs.

Sorbonne. 